\section{Hypotension induced by interleukin-3 in patients on angiotensin-converting enzyme inhibitors}

SIR - The use of haematopoietic growth factors is increasing. It is, therefore, essential to demonstrate their efficacy and safety when given with other commonly prescribed medicines. We treated 26 patients with ovarian or small-cell undifferentiated cancers with chemotherapy followed by recombinant human interleukin-3 (rhIL-3) $(0 \cdot 125-7 \cdot 5 \mu \mathrm{g} / \mathrm{kg}$ daily). ${ }^{1} 3$ of these patients were also receiving angiotensinconverting enzyme (ACE) inhibitors for hypertension. All 3 developed marked hypotension (WHO toxicity grade 2 or 3) within 1 4 hours of the first rhIL-3 injection. In all 3 patients discontinuation of the $\mathrm{ACE}$ inhibitor while continuing rhIL-3 resulted in return of the blood pressure to normal. After stopping rhIL-3 the blood pressure increased and ACE inhibitors were again required. Hypotension was not observed in 22 patients given rhIL-3 without ACE inhibitors. 1 other patient, not receiving an ACE inhibitor, became hypotensive during a period of neutropenic fever. The fever resolved with antibiotic therapy.

The hypotensive effects of ACE inhibitors are mediated by inhibiting the conversion of angiotensin I into angiotensin II (a powerful vasoconstrictor). Additionally, inhibition of kinase II reduces the breakdown of bradykinin, resulting in vasodilation. ${ }^{2}$ Furthermore, rhIL-3 stimulates the synthesis of secondary cytokines (eg, IL-6) ${ }^{3}$ and histamine. ${ }^{4}$ The hypotension induced by these substances is mediated via nitric oxide (NO). Both bradykinin and histamine stimulate rapid (within minutes) NO production by activation of constitutive NO synthase. Cytokines, including IL-6, cause a late and more sustained NO generation via an inducible NO synthase found in endothelium and vascular smooth muscle. 5

The urine from 1 of the hypotensive patients was assayed for nitrate and cGMP, as indicators of in-vivo NO production. In the first chemotherapy cycle without rhIL-3 the 24-hour urinary nitrate and cGMP excretion did not exceed $1 \mathrm{mmol}$ and $1 \mu \mathrm{mol}$, respectively. In the second cycle with rhIL-3, 24-hour nitrate excretion rose from $0.9 \mathrm{mmol}$ before chemotherapy and $0.8 \mathrm{mmol}$ before rhIL-3 to $5 \cdot 7$ mmol on day 5 of rhIL-3 administration. cGMP rose from $0.9 \mu \mathrm{mol}$ to $2.6 \mu \mathrm{mol}$ on day 5 . In another 16 patients, not receiving ACE inhibitors and not developing hypotension, 24-hour nitrate was measured in 9 and 26 cycles of chemotherapy with and without rhIL-3, respectively. In none of these cycles did the 24-hour nitrate excretion exceed $1.3 \mathrm{mmol}$ (control cycles 0.7 [SE 0.3$] \mathrm{mmol}$; cycles with rhIL-3 $0 \cdot 8[0 \cdot 3] \mathrm{mmol})$.

These data suggest that NO generated in vessel walls by bradykinin and histamine (via constitutive NO synthase) and secondary cytokines (via inducible NO synthase) were responsible for the increase of urinary nitrate and cGMP. Without priming by ACE inhibitors, the effects of rhIL-3 were too small to lower blood pressure or could easily be compensated by counter-regulatory mechanisms. We suggest that synergism between forces acting to generate $\mathrm{NO}$, in the absence of the counteracting effects of angiotensin II, were responsible for the rapid induction of hypotension in patients treated with ACE inhibitors combined with rhIL-3.

\section{MW Dercksen, K Hoekman, J J Visser,}

\section{WW ten Bokkel Huinink, H M Pinedo, *J Wagstaff}

European Cancer Centre, Amsterdam; *Department of Oncology, Free University Hospital, 1081 HV Amsterdam, the Netherlands; Department of Surgery, Free University Hospital; and Department of Medical Oncology, the Netherlands Cancer Instıtute/Antonı van Leeuwenhoekhuis, Amsterdam
1 Dercksen MW, Hoekman K, ten Bokkel Huinink WW, et al. Effects of interleukin-3 on myelosuppression induced by chemotherapy for ovarian cancer and small cell undifferentiated tumours. $\mathrm{Br} F \mathrm{Cancer}$ 1993; 68: 996-1003.

2 Félétou $M$, Germain $M$, Teisire B. Converting-enzyme inhibitors potentiate bradykinin-induced relaxation in vitro. Am $\mathcal{F}$ Physiol 1992; 262: H838-45.

3 Lindemann A, Ganser A, Herrmann F, et al. Biological effects of recombinant interleukin-3 in vivo. $f$ Clin Oncol 1991; 9: 2120-27.

4 Dy M, Schneider E. Interleukin-3 (IL-3) and granulocyte-macrophage colony stimulating factor: two cytokines increasing histamine synthesis by hematopoiëtic cells. Eur Cytokine Netw 1991; 2: 153-72.

5 Moncada S, Palmer RM, Higgs EA. Nitric oxide: physiology, pathophysiology, and pharmacology. Pharmacol Rev 1991; 43: 109-42.

\section{Nitric oxide in ulcerative colitis}

SIR-Lundberg and colleagues (Dec 17, p 1673) suggest that nitric oxide (NO) concentrations in the colonic lumen might be increased in ulcerative colitis. The chemiluminescence method used by these workers depends on the release of energy from the reaction of NO with ozone, and is therefore not truly direct. Ozone is a reactive species and the colonic lumen contains many gaseous substances, so there is doubt as to its specificity under these conditions. $\mathrm{H}_{2} \mathrm{~S}$ in particular may react with ozone to produce chemiluminescent species, and is known to be raised in ulcerative colitis.

We have evidence that the concentration of NO might be increased in ulcerative colitis, with a molecule-specific technique. The rectal lumen in 8 patients with active disease and 8 controls was perfused for $1 \mathrm{~h}$ with a nitrogen stream, and $\mathrm{NO}$ was collected in a cold trap at $-196^{\circ} \mathrm{C}$. The NO thus obtained was measured by infrared diode laser spectroscopy. This technique allows high detection sensitivity and specificity and we have checked that the following gases have no absorptions overlapping the NO transitions used in the measurements: $\mathrm{H}_{2} \mathrm{O}, \mathrm{H}_{2} \mathrm{~S}, \mathrm{CH}_{4}, \mathrm{CO}$, $\mathrm{CO}_{2}, \mathrm{~N}_{2} \mathrm{O}, \mathrm{NO}_{2}, \mathrm{~N}_{2} \mathrm{O}_{3}, \mathrm{~N}_{2} \mathrm{O}_{4}$. The estimated minimum detectable amount of $\mathrm{NO}$ was $0.1 \mathrm{nmoL}$. NO was detected in 4 of 8 patients with active ulcerative colitis but in none of the controls $(p<0.05)$. The amounts measured varied from $0 \cdot 13$ to $1 \cdot 1 \mathrm{nmoL}$. Patients negative for $\mathrm{NO}$ all had blood visible in the rectum and the collection tubing.

NO is avidly bound by haemoglobin and we suspect that this was why we could not detect it in 4 of our patients. If the technique used by Lundberg and co-workers were truly specific for NO, we would have expected them to encounter the same difficulty. Nevertheless, Lundberg and colleagues' work supports our observation that NO synthesis is increased in ulcerative colitis, ${ }^{1}$ a finding that could prove to be of both pathogenetic and therapeutic importance.

* P D Reynolds, $S J$ Middleton, G M Hansford, $J$ O Hunter *Gastroenterology Research Unit, Addenbrooke's Hospital, Cambridge CB2 2QQ, UK; and Department of Chemistry, University of Cambridge

1 Middleton SJ, Shorthouse M, Hunter JO. Increased nitric oxide synthesis in ulcerative colitis. Lancet $1993 ; 341$ : 465-66.

SIR-Lundberg and colleagues' study complements our own investigation showing raised serum concentrations of NO metabolites in 26 patients with severe attacks of ulcerative colitis. ${ }^{1}$ However, in that study concentrations of C-reactive protein were shown to predict clinical outcome more accurately than NO metabolites. Lundberg's data concern us on several counts.

Only 6 patients were investigated, on one occasion only. Lundberg and co-workers did not correlate NO concentrations with disease activity or response to medical therapy. Without this information, their attempts to draw conclusions about the effects of corticosteroids on NO 